\title{
Enhancing synergy of CAR $T$ cell therapy and oncolytic virus therapy for pancreatic cancer.
}

\author{
Rachel Walker, Pedro E. Navas, Samuel H. Friedman, Simona Galliani, Aleksandra Karolak, Fiona \\ Macfarlane, Robert Noble, Jan Poleszczuk, Shonagh Russell, Katarzyna A. Rejniak, Amir Shahmoradi, \\ Frederik Ziebell, Jason Brayer ${ }^{*}$, Daniel Abate-Daga ${ }^{*}$, and Heiko Enderling ${ }^{*}$
}

\begin{abstract}
The poor immunogenicity of pancreatic tumors makes them particularly difficult to treat. Standard chemotherapies and single agent immunotherapies have had notoriously little success in this arena. Oncolytic virus therapy has the potential to enhance the penetration of immunotherapeutically-delivered CAR $T$ cells into the tumor and improve treatment outcomes. We evaluate this potential by combining two different mathematical approaches: an ordinary differential equation model to simulate population level tumor response to cytotoxic activity of $T$ cells, coupled with an agentbased model to simulate the enhancement of CAR $T$ cell penetration by oncolytic virus therapy.
\end{abstract}

\section{BACKGROUND}

Pancreatic cancer is the fourth most common cause of cancer-related death, with 5 -year survival rates of only $7 \%^{[1]}$. This poor prognosis is, at least in part, due to the insufficient immunogenicity of pancreatic tumors ${ }^{[2]}$. In recent years, an increasing amount of attention has been given to gene therapy technologies designed to "train" the patient's immune system to detect and kill cancer cells. This novel approach has the potential to overcome treatment challenges attributable to the strong immunosuppressive measures imposed by both the tumor cell and the stromal microenvironment.

Chimeric antigen receptor (CAR) technology involves the retroviral insertion of genes into primary human lymphocytes, which introduces a highly specific antigen receptor to both direct the cognate anti-tumor interaction and provide the necessary activation signals to elicit a potent tumor-killing response ${ }^{[3]}$. The genetically engineered T cells can be activated ex-vivo by antibody-mediated CD3 and CD28 and further expanded massively in culture with

Research supported by H. Lee Moffitt Cancer Center \& Research Institute.

R. W, P. E. N., A. K., J. P., S. R., K. A. R., A. R. A. A., J. B., D. A.-D., H. E. are with the H. Lee Moffitt Cancer Center \& Research Institute, Tampa, FL 33612 USA (813-745-3562; fax: 813-745-6497; e-mail: \{Jason.Brayer;Daniel.AbateDaga;Heiko.Enderling\}@moffitt.org).

S. F. Author is with the Center for Applied Molecular Medicine, University of Southern California, Los Angeles, CA 90033 USA

S. G. Author is with the Stuttgart Research Center Systems Biology (SRCSB), 70569 Stuttgart, Germany

F. M. Author is with the School of Mathematics and Statistics, University of St Andrews, KY16 9SS UK

R. N. Author is with the Institut des Sciences de l'Evolution, University of Montpellier, Montpellier 34095, France

A. S. Author is with the Institute for Computational Engineering and Sciences, The University of Texas at Austin, Austin, TX 78712 USA

F. Z. Author is with the Institute of Applied Mathematics and the German Cancer Research Center (DKFZ), Heidelberg, Germany cytokines prior to re-infusion into the patient to mount their attack on the cancer cell population. CAR T cell therapy has demonstrated great promise in both the laboratory and the clinical setting $^{[4]}$.

Despite this promise, extravasation of CAR $\mathrm{T}$ cells into solid tumors is limited due to the lack of homing cues for ex vivo activated T cells, resulting in only an estimated $0.3 \%$ of infused cells effectively reaching the tumor ${ }^{[5]}$. Moreover, in pancreatic cancer the presence of fibrotic stroma presents a physical barrier to $\mathrm{T}$ cells, impeding their infiltration ${ }^{[6]}$. Improving CAR $\mathrm{T}$ cell penetration into the tumor is crucial for increasing treatment efficacy, and may be achievable with complementary local therapy. In situ injection of oncolytic viruses has the potential to debulk the tumor and increase stroma permeability for deeper infiltration of CAR $\mathrm{T}$ cells at the tumor site, with an additional possibility of further $\mathrm{T}$ cell recruitment from the vasculature ${ }^{[7]}$. Attenuated strains of the measles RNA virus have been used for vaccination; oncolytic measles virotherapy has been found to cause substantial death of tumor cells by initial infection and subsequent viral replication within infected cells ${ }^{[8,9]}$.

The augmentation of localized $\mathrm{T}$ cell recruitment through oncolytic virus therapy requires the determination of synergy mechanisms. The overall goal of this project is therefore to derive mathematically and confirm experimentally optimal sequencing of oncolytic virus therapy with CAR $\mathrm{T}$ cell therapy. Because of the complex dynamics that govern these interactions at different biological scales, this project is necessarily multiscale and multidisciplinary.

\section{ILLUSTRATIVE RESUltS OF APPLICATION OF METHODS}

The first component of the study is the development of a non-spatial, compartmental mathematical model of the dynamics of the respective populations of cancer cells, CAR $\mathrm{T}$ cells within the tumor, and CAR T cells circulating in the blood using a system of ordinary differential equations (ODEs). Circulating $\mathrm{T}$ cells extravasate into the tumor site where they have the capacity to induce the death of cancer cells until they become exhausted or deactivated.

To represent the influence of oncolytic virus therapy, the cancer cell population was segregated into those infected and those uninfected, with an initial instantaneous transfer of cells into the infected compartment to represent viral injection. When infected, cells undergo death by lysis which can in turn release virus particles from the lysing cell, continuing the spread of the virus through the tumor population. With the addition of oncolytic virus therapy, a reduction in the total tumor cell population was observed; the model predicts synergistic effects of a combination CAR 

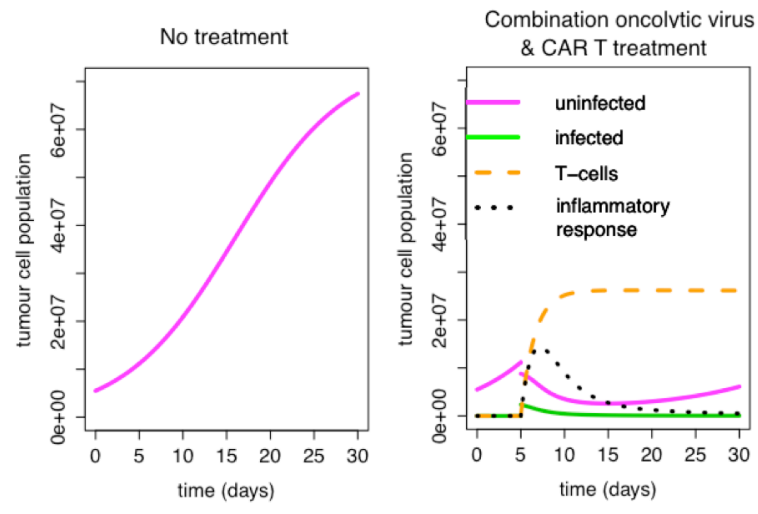

Figure 1. Population dynamics for untreated tumor cells (left) and respective uninfected and infected tumor cells under the influence of synergistic oncolytic virus and CAR T cell therapy (right).

$\mathrm{T}$ cell and oncolytic virus therapy, resulting in greater effectiveness than both monotherapies independently (example output demonstrated in Fig. 1).

To complement the ODE model, a high-resolution, spatial agent-based model (ABM) was developed that accounts for tumor-CAR T cell interactions and perturbation by oncolytic viruses. We determined the spatial distribution of stroma and cancer cells by discretizing a tissue pathology image using Image ${ }^{[10]}$. The model was able to demonstrate the extravasation of CAR $\mathrm{T}$ cells into the tumor site from the defined blood vessels, the initial injection of the oncolytic virus at a predefined tumor site, and the subsequent death of infected cancer cells and corresponding virus spreading. The model allowed simple quantification of the decrease in cancer cell numbers and is configurable for both oncolytic virus and CAR $\mathrm{T}$ cell injection timing (an example output can be found in Fig. 2).

\section{Quick Guide to The Methods}

\section{A. Equations}

$$
\begin{aligned}
& \frac{d C_{i}}{d t}=-v C_{i}-a T \frac{C_{i}}{C_{i}+C_{u}}(1+e F)+u v C_{i} \frac{C_{u}}{C_{i}+C_{u}} \\
& \frac{d C_{u}}{d t}=\lambda C_{i}\left(1-C_{i} / K\right)-a T \frac{C_{u}}{C_{i}+C_{u}}(1+e F)-u v C_{i} \frac{C_{u}}{C_{i}+C_{u}} \\
& \frac{d T}{d t}=q T_{r}-m T \quad \text { (3) } \quad \frac{d T_{r}}{d t}=-q T_{r} \quad \text { (4) } \quad \frac{d F}{d t}=b v C_{i}-n F
\end{aligned}
$$

Equations (1) to (5) comprise the ODE model for respective populations of virus-infected cells $\left(C_{i}\right)$, uninfected cells $\left(C_{u}\right)$, T cells in the tumor $(T)$ and $\mathrm{T}$ cells circulating in the blood $\left(T_{r}\right)$, and the overall inflammatory response $(F)$. In (1), the first term on the RHS represents the death of infected cells due to lysis, followed by the $\mathrm{T}$ cell killing of cancer cells at a rate proportional to both their interaction rate and the extent of the inflammatory response. The rate of infection of uninfected cells is represented by the last term in (1) and is proportional to the death by lysis. Only the uninfected cancer cells proliferate; growth behavior of the tumor population is obtained from experimental literature and is dependent upon a carrying capacity $K$. Cells leave the uninfected compartment when they are either killed by $\mathrm{T}$ cells or become infected, as previously described. The initial in situ

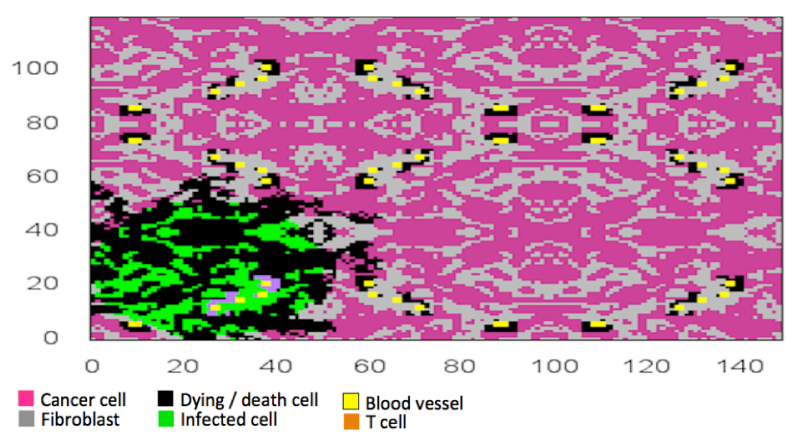

Figure 2. Simulation snapshot of ABM. Note dying cancer cells due to both the oncolytic virus and CAR T cells spreading from the blood vessels.

virus injection is represented by an instantaneous shift in a subpopulation of the uninfected cancer cells to the infected cell compartment.

$\mathrm{T}$ cells in the tumor arrive due to extravasation from the blood vessels, and interact with the cancer cells until they become deactivated or exhausted, represented mathematically in (3). The $\mathrm{T}$ cell reservoir in the blood is defined by an initial condition representing the instantaneous CAR $\mathrm{T}$ cell injection, at which point these activated $\mathrm{T}$ cells circulate in the blood and extravasate into the tumor at a fixed rate proportional to their population (4). Finally, in (5), the inflammatory response increases due to death by lysis, and naturally decays.

The model features 8 free parameters, assuming $\lambda$ and $K$ the growth rate and carrying capacity of the tumor are derivable from the experimental data. These parameters are the rate of death by lysis (v), the rate of $\mathrm{T}$ cell killing of cancer cells $(a)$, the strength of dependence of T cell killing on the inflammatory response $(e)$, the infection rate of uninfected cells $(u)$, the infiltration rate of $T$ cells from the blood $(q)$, the $\mathrm{T}$ cell exhaustion rate $(m)$, the dependence of the inflammatory response on death by lysis $(b)$, and the rate of decay of the inflammatory response $(n)$. While several of these parameters may be obtainable from the experimental literature, further study will allow parameter sensitivity analysis and potential simplification of the model.

Both preliminary models demonstrated the ability to identify conditions for optimizing tumor cell population reduction in combination $\mathrm{CAR} \mathrm{T}$ cell and oncolytic virus therapies for pancreatic cancer. In future work, we need to more accurately parameterize and calibrate these models to generate robust suggestions for the optimization of treatment protocols. The synergistic outcomes simulated by these models may ultimately be tested in a clinical trial.

\section{ACKNOWLEDGMENT}

We would like to thank Dr. Alexander R. A. Anderson and the Moffitt Cancer Center for organization and support of the 5th Annual Integrated Mathematical Oncology workshop, Immune Cancer, where this project was conceived.

\section{REFERENCES}

1. Siegel RL, Miller KD, Jemal A. Cancer statistics, 2015. CA Cancer J Clin. 2015;65: 5-29. doi:10.3322/caac.21254 Clark CE, Hingorani SR, Mick R, Combs C, Tuveson DA, 
bioRxiv preprint doi: https://doi.org/10.1101/055988; this version posted May 30, 2016. The copyright holder for this preprint (which was not

Vonderheide RH. Dynamics of the immune reaction to pancreatic cancer from inception to invasion. Cancer Res. American Association for Cancer Research; 2007;67: 9518-9527. doi:10.1158/0008-5472.CAN-07-0175

3. Abate-Daga D, Rosenberg SA, Morgan RA. Pancreatic cancer: Hurdles in the engineering of CAR-based immunotherapies. Oncoimmunology. 2014;3: e29194. doi:10.4161/onci.29194

4. Feldman SA, Assadipour Y, Kriley I, Goff SL, Rosenberg SA. Adoptive Cell Therapy--Tumor-Infiltrating Lymphocytes, T-Cell Receptors, and Chimeric Antigen Receptors. Semin Oncol. 2015;42: 626-639. doi:10.1053/j.seminoncol.2015.05.005

5. Moon EK, Carpenito C, Sun J, Wang L-CS, Kapoor V, Predina J, et al. Expression of a functional CCR2 receptor enhances tumor localization and tumor eradication by retargeted human $\mathrm{T}$ cells expressing a mesothelin-specific chimeric antibody receptor. Clin Cancer Res. American Association for Cancer Research; 2011;17: 4719-4730. doi:10.1158/1078-0432.CCR-11-0351

6. Bhaw-Luximon A, Jhurry D. New avenues for improving pancreatic ductal adenocarcinoma (PDAC) treatment: Selective stroma depletion combined with nano drug delivery. Cancer Lett. 2015;369: 266-273. doi:10.1016/j.canlet.2015.09.007

7. Nishio N, Dotti G. Oncolytic virus expressing RANTES and IL15 enhances function of CAR-modified T cells in solid tumors. Oncoimmunology. 2015;4: e988098.

8. Galanis E, Hartmann LC, Cliby WA, Long HJ, Peethambaram PP, Barrette BA, et al. Phase I trial of intraperitoneal administration of an oncolytic measles virus strain engineered to express carcinoembryonic antigen for recurrent ovarian cancer. Cancer Res. 2010;70: 875-882. doi:10.1158/0008-5472.CAN-09-2762

9. Heinzerling L, Künzi V, Oberholzer PA, Kündig T, Naim H, Dummer R. Oncolytic measles virus in cutaneous T-cell lymphomas mounts antitumor immune responses in vivo and targets interferon-resistant tumor cells. Blood. 2005; 106: 22872294. doi:10.1182/blood-2004-11-4558

10. Abramoff MD, Magalhães PJ, Ram SJ. Image processing with ImageJ. Biophotonics international. Laurin Publishing; 2004. pp. $36-42$. 